\title{
Robust Control for Magnetostrictive Actuators
}

\author{
Akira Yonenaga* and Xinkai Chen** \\ *Graduate School of Engineering and Science, \\ Shibaura Institute of Technology University, Saitama City, Saitama, Japan \\ **Department of Electronic Information System, \\ Shibaura Institute of Technology University, Saitama City, Saitama, Japan
}

Email:mf15082@shibaura-it.ac.jp

\begin{abstract}
The hysteresis phenomenon exists in magnetostrictive actuators. When the hysteresis nonlinearity exists in a controlled system, the system usually exhibits inaccuracies or oscillations and even instability due to the undifferentiable and nonmemoryless character of the hysteresis. This paper proposes the robust control in broadband for magnetostrictive actuators, where the hysteresis is described by the Preisach model.
\end{abstract}

\section{Introduction}

Magnetostrictive materials, which are alloys consisted of rare-earth and iron, have been received much attention recently due to their possible deformation when a magetostric field is applied from outside. Based on this property, the magnetostrictive materials are used in actuators which are called "magnetostrictive actuators" to convert the electric energy into mechanical energy. These kinds of actuators are used to meet the requirement of nanometer resolution in displacement, high stiffness and rapid response. Furthermore, the magnetostrictive actuators are possible to be driven by low voltage. For these reasons, the magnetostrictive actuators are expected to replace other existing actuators to achieve the abovementioned requirements. However, the main disadvantage of the magnetostrictive actuators is that they are very difficult to control because of the hysteresis phenomenon existing between the applied current and the displacement.

This paper will discuss the robust control problem of the magnetostrictive actuators, where Terfenol-D (which is a with composite of $\mathrm{Tb}_{0.3} \mathrm{Dy}_{0.7} \mathrm{Fe}_{2}$ ) type actuators are taken into consideration because, unlike other types of magnetostrictive actuators, Terfenol-D type actuators do not need cryogenic temperature environments and they can work in room temperature environments.

In this paper, Section 2 gives the hysteresis model. Section 3 presents the basic control method, and the experimental results are given in Section 4. In Section 5, the improvement of the hysteresis model and control design are considered and are supported by experimental results. Section 6 concludes this paper.

\section{Hysteresis model}

Hysteresis is a nonlinear phenomenon that the present outputs depends on not only the present input but also the past inputs. That is to say, it is a phenomenon that the outputs for a certain input may be different.

There are several mathematical model which can express hysteresis. Among those models, the Preisach model is of higher ability to describe the hysteresis than other models [3], [4], [7], [13].

In this paper, the input-output relation of magnetostrictive actuator is expressed by Preisach model. This model is composed by relay operators and density functions. The outputs of relay operators are \pm 1 with threshold $\alpha$ and $\beta$. Fig. 2 shows the input and output relation of a relay operator.

Assume $w(k)=\gamma_{\alpha \beta}[v](k)$. The behavior of the relay operator is defined as follows. Also the initial setting of $w(k)$ is $w_{-1} \in\{-1,1\}$.

$$
w(k)=\left\{\begin{array}{cl}
-1 & \text { if } v(k)<\beta \\
1 & \text { if } v(k)>\alpha \\
w(k-1) & \text { if } \beta \leq v(k) \leq \alpha
\end{array}\right.
$$
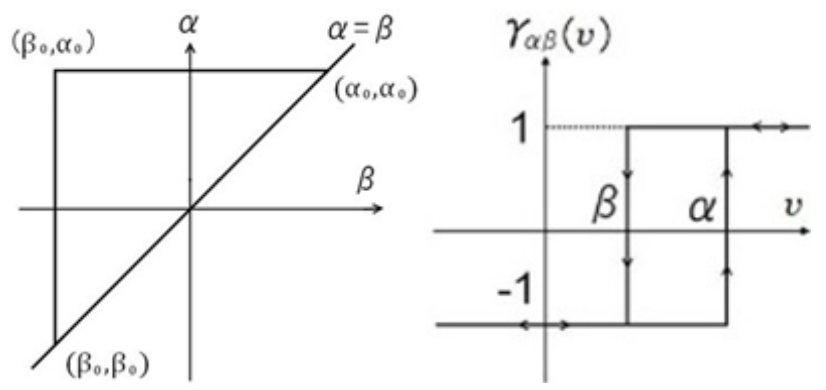

Fig.1 Integral calculus domain. Fig.2 Relay operator.

Fig. 1 shows the variation domain of the thresholds, where $\alpha_{0}$ and $\beta_{0}$ are sufficient large values. Also, Fig. 3 
shows the variation of integral calculus domain, where a certain input signal is consider in Preisach model. From Fig. 3 , it can be seen that the integral calculus domain varies when the input $v(k)$ changes its values, where $S^{+}(\mathrm{k})$ shows the value of the relay operator takes $+1, S^{-}(\mathrm{k})$ shows the value of the relay operator takes -1 . Memory characteristics of the hysteresis is expressed by the stairs.

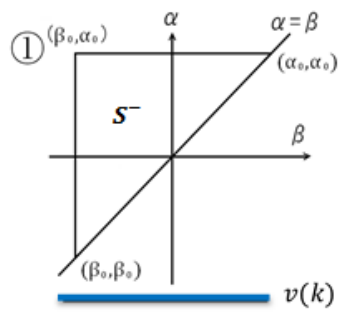

(4)
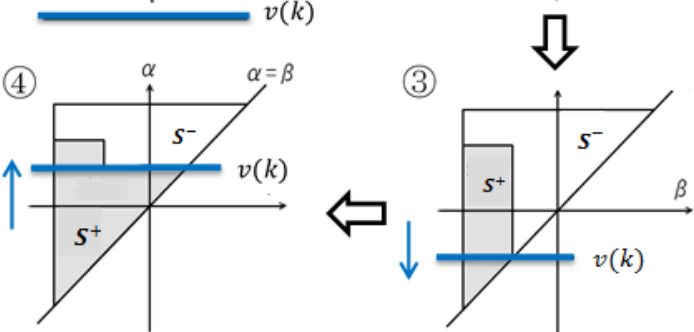

(5)
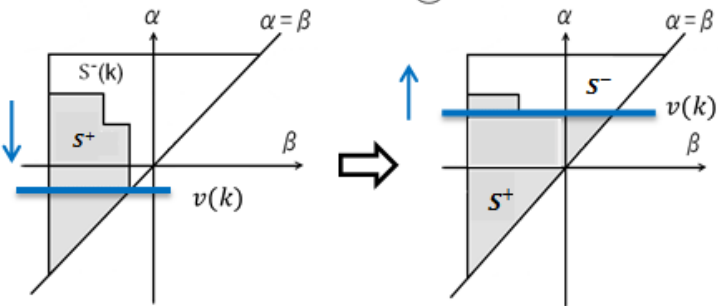

Fig. 3 The variation of integral calculus domain.

Preisach model is mathematically defined as

$$
u(k)=\iint_{\beta \leq \alpha} \mu(\beta, \alpha) \gamma_{\alpha \beta}[v](k) d \beta d \alpha,
$$

where $\mu(\beta, \alpha)$ are unknown parameters.

Fig. 4 shows the simulation result of the Preisach model. Fig. 5 shows the measured input-output relation of a magnetostrictive actuator. From Fig. 4 and Fig. 5, it can be seen that Preisach model has the ability to describe the hysteresis behavior in magnetostrictive actuator.

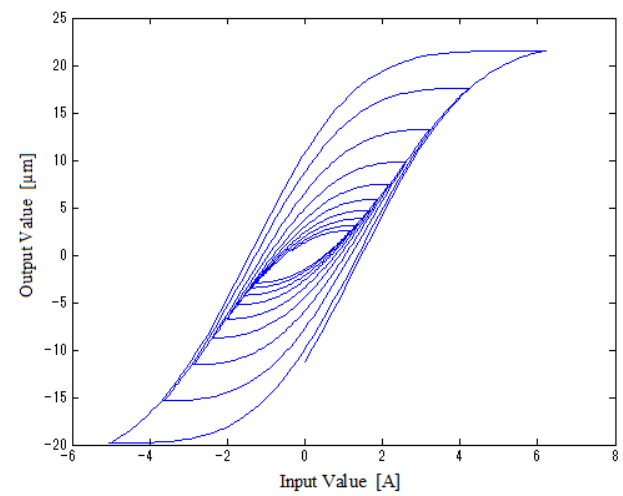

Fig. 4 Simulation result.

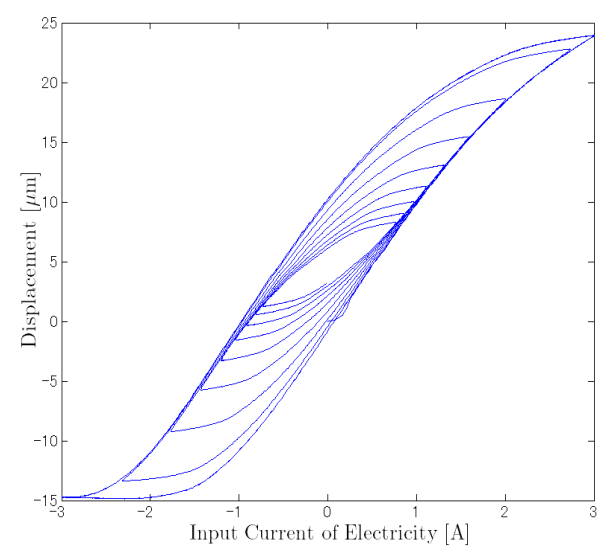

Fig. 5 Experimental result.

\section{Design of the control technique}

This section considers the control technique for the actuator with hysteresis. Control formulation is constructed in discrete time, where $\mathrm{k}$ is used to express the time instant.

\subsection{Adaptive control}

In order to calculate the output corresponding to a given input, it is necessary to use the value of density function. However density function is usually unknown. This problem is solved by using adaptive method [1], [2], [5], [6], [12]. Suppose the estimate of $\mu(\beta, \alpha)$ at instant $\mathrm{k}$ is $\hat{\mu}(\beta, \alpha, k)$. By defining the estimated output at instant $\mathrm{k}$

$$
\hat{u}(k)=\iint_{\beta \leq \alpha} \hat{\mu}(\beta, \alpha, k) \gamma_{\alpha \beta}[v](k) d \beta d \alpha
$$

and output error

$$
e(k)=u(k)-\hat{u}(k),
$$

the density function is updated online by

$$
\hat{\mu}(\beta, \alpha, k)=\left|\hat{\mu}(\beta, \alpha, k-1)+\frac{\rho}{S} e(k-1) \gamma_{\alpha \beta}[v](k-1)\right|
$$

where $\rho$ is adaptation gain $(0<\rho<2)$, $S$ is the integral area. It should be noted that $\hat{\mu}(\beta, \alpha, \mathrm{k})$ is not negative. Also, initial values of $\hat{\mu}(\beta, \alpha, k)$ are assumed non-negative.

Next, the estimation error of the density function is define as

$$
\tilde{\mu}(\beta, \alpha, k)=\hat{\mu}(\beta, \alpha, k)-\mu(\beta, \alpha)
$$

Taking the difference of $\iint_{\beta \leq \alpha} \tilde{\mu}^{2}(\beta, \alpha, k) d \beta d \alpha$ at k-th step and (k-1)-th step yields

$$
\begin{aligned}
& \iint_{\beta \leq \alpha} \tilde{\mu}^{2}(\beta, \alpha, k) d \beta d \alpha-\iint_{\beta \leq \alpha} \tilde{\mu}^{2}(\beta, \alpha, k-1) d \beta d \alpha \\
\leq & \iint_{\beta \leq \alpha}\left\{\hat{\mu}^{\prime}(\beta, \alpha, k)-\mu(\beta, \alpha)\right\}^{2} d \beta d \alpha \\
- & \iint_{\beta \leq \alpha} \tilde{\mu}^{2}(\beta, \alpha, k-1) d \beta d \alpha
\end{aligned}
$$




$$
\begin{aligned}
& =2 \frac{\rho}{S} e(k-1) \iint_{\beta \leq \alpha} \gamma_{\alpha \beta}[v](k-1) \tilde{\mu}(\beta, \alpha, k-1) d \beta d \alpha \\
& +\frac{\rho^{2}}{S^{2}} e^{2}(k-1) \iint_{\beta \leq \alpha} \gamma_{\alpha \beta}[v](k-1) \gamma_{\alpha \beta}[v](k-1) d \beta d \alpha \\
& =-2 \frac{\rho}{S} e_{1}^{2}(k-1)+\frac{\rho^{2}}{S} e_{1}{ }^{2}(k-1) \\
& =-\frac{\rho(2-\rho)}{S} e^{2}(k-1)
\end{aligned}
$$

Now, summing up the relation (6) from $\mathrm{k}=1$ to $\mathrm{k}=\mathrm{n}$ gives

$$
\begin{aligned}
& \iint_{\beta \leq \alpha} \tilde{\mu}^{2}(\beta, \alpha, n) d \beta d \alpha-\iint_{\beta \leq \alpha} \tilde{\mu}^{2}(\beta, \alpha, 0) d \beta d \alpha \\
& \leq-\frac{\rho(2-\rho)}{S} \sum_{k=1}^{n} e^{2}(k-1)
\end{aligned}
$$

From Equation (7), it can be seen that $\iint_{\beta \leq \alpha} \tilde{\mu}^{2}(\beta, \alpha, k) d \beta d \alpha$ is bounded and $\lim _{\mathrm{k} \rightarrow \infty} e(k)=0$.

\subsection{Calculation of the estimated output}

Since the calculation of $\hat{u}(k)$ in Equation (2) is very complicated, we try to simply it by using the available value of $\hat{u}(k-1)$. For this purpose, Equation (8) is derived from Equation (2) and Equation (4).

$$
\begin{aligned}
\hat{u}(k)= & \iint_{\alpha \geq \beta} \hat{\mu}(\beta, \alpha, k) \gamma_{\beta \alpha}[v](k) d \beta d \alpha \\
=\hat{u}(k-1)+ & 2 \iint_{S_{3}} \hat{\mu}(\beta, \alpha, k-1) \gamma_{\beta \alpha}[v](k) d \beta d \alpha \\
& +\frac{\rho}{S} e(k-1)\left\{S-2\left(s_{3} \text { area }\right)\right\}
\end{aligned}
$$

where $\hat{u}(k-1)$ is the estimate output at $(\mathrm{k}-1)$-th step. Also, $s_{3}$ shows the area of the region where the value of the relay operator changes from +1 to -1 , or from -1 to +1 . The area in the slanted line is $s_{3}$ in Fig. 6 .

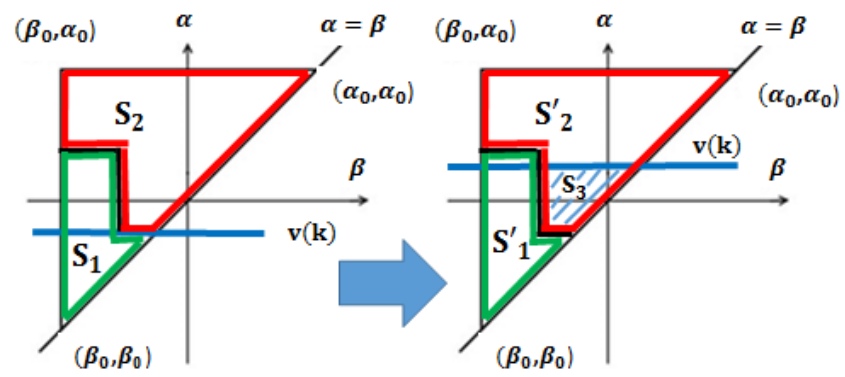

Fig. 6 Variation of the integral domains taking +1 or -1 .

Fig 6 shows the variation of the integral domains when the input increases, where $S_{1}$ and $S_{1}^{\prime}$ show the region that the value of relay operator takes $+1, S_{2}$ and $S^{\prime}{ }_{2}$ show the region that the value of relay operator takes -1 , and $S_{3}$ shows the region that the value of relay operator changes from +1 to -1 .

Thus, the estimated output is calculated by only focusing on the region where the value of the relay operator changes because $\hat{u}(k)$ can be expressed by using $\hat{u}(k-1)$ in
Equation (8). Such method makes the calculation processing simpler.

\subsection{Input calculation}

Now, let us consider Equation (9), where $u_{d}$ is the desired output.

$$
u_{d}(k)=\iint_{\beta \leq \alpha} \hat{\mu}(\beta, \alpha, k) \gamma_{\alpha \beta}\left[v^{*}\right](k) d \beta d \alpha
$$

$v^{*}(k)$ can be calculated by satisfying Equation (9). This calculated value is the input which should be applied to the magnetostrictive actuator.

As a matter of fact, finding a $v^{*}(k)$ such that relation (9) holds is very difficult practically. For that purpose, let as consider the following weak relation

$\left|u_{d}(k)-\iint_{\alpha \geq \beta} \hat{\mu}(\beta, \alpha, k) \gamma_{\beta \alpha}\left[v^{*}\right](k) d \beta d \alpha\right| \leq \delta$

where $\delta$ is the admissible parameter. The algorithm of calculating input value is as follows, where the interpolation method is used.

Step $1: v^{(0)}(k):=v^{*}(k-1), l:=0$.

Step $2: u^{(l)}(k):=\iint_{\alpha \geq \beta} \hat{\mu}(\beta, \alpha, k) \gamma_{\beta \alpha}[v](k) d \beta d \alpha$

If $\left|u^{(l)}(k)-u_{d}\right| \leq \delta$, go to Step 6;

$$
\begin{gathered}
\text { Else if } u^{(l)}(k)<u_{d}(k)-\delta \text {, let } v^{(l+1)}(k) \\
:=v^{(l)}(k)+\Delta
\end{gathered}
$$

and $l:=l+1$, then go to Step 3 .

Else $\left(\right.$ i.e. $\left.u^{(l)}(k)>u_{d}(k)+\delta\right)$, let

$$
\begin{aligned}
v^{(l+1)}(k) & :=v^{(l)}(k)-\Delta \text { an } \\
& :=l+1, \text { then go to Step } 4 .
\end{aligned}
$$

Step $3: u^{(l)}(k):=\iint_{\alpha \geq \beta} \hat{\mu}(\beta, \alpha, k) \gamma_{\beta \alpha}[v](k) d \beta d \alpha$ If $\left|u^{(l)}(k)-u_{d}(k)\right| \leq \delta$, go to Step 6;

Else if $u^{(l)}(k)<-\delta$, let $v^{(l+1)}(k):=v^{(l)}(k)+\Delta$ and $l:=l+1$, then go to Step 3 .

Else $\left(\right.$ i.e. $\left.u^{(l)}(k)>u_{d}+\delta\right)$, let $\underline{v}^{(l)}=v^{(l-1)}(k)$ and $\bar{v}^{(l)}:=v^{(l)}(k)$, then go to Step 5 .

Step $4: u^{(l)}(k):=\iint_{\alpha \geq \beta} \hat{\mu}(\beta, \alpha, k) \gamma_{\beta \alpha}[v](k) d \beta d \alpha$ If $\left|u^{(l)}(k)-u_{d}\right| \leq \delta$, go to Step 6;

Else if $u^{(l)}(k)<u_{d}-\delta$, let $v^{(l+1)}(k):=v^{(l)}(k)-\Delta$ and $l:=l+1$, then go to Step 3 .

Else (i.e. $\left.u^{(l)}(k)<u_{d}-\delta\right)$, let $\underline{v}^{(l)}=v^{(l)}(k)$ and $\bar{v}^{(l)}:=v^{(l-1)}(k)$, then go to Step 5 .

Step $5: \underline{u}^{(l)}(k):=\iint_{\beta \leq \alpha} \hat{\mu}(\beta, \alpha, k) \gamma_{\alpha \beta}\left[\underline{v}^{(l)}\right](k) d \beta d \alpha$,

$$
\begin{gathered}
\bar{u}^{(l)}(k):=\iint_{\beta \leq \alpha} \hat{\mu}(\beta, \alpha, k) \gamma_{\alpha \beta}\left[\bar{v}^{(l)}\right](k) d \beta d \alpha, v^{(l+1)}(k) \\
:=\underline{v}^{(l)}(k)+\left(\bar{v}^{(l)}(k)-\underline{v}^{(l)}(k)\right) \frac{u^{(l)}(k)-\underline{u}^{(l)}(k)}{\bar{u}^{(l)}(k)-\underline{u}^{(l)}(k)} \\
\text { Let } l:=l+1 \text { and }
\end{gathered}
$$




$$
u^{(l)}(k):=\iint_{\alpha \geq \beta} \hat{\mu}(\beta, \alpha, k) \gamma_{\beta \alpha}[v](k) d \beta d \alpha
$$

If $\left|u^{(l)}(k)-u_{d}\right| \leq \delta$, go to Step 6;

Else if $u^{(l)}(k)<u(k)-\delta$, let $v^{(l+1)}(k):=v^{(l)}(k)+\Delta$ and $\bar{v}^{(l)}:=v^{(l-1)}(k)$, then go to Step 5 ;

Else $\left(\right.$ i.e. $\left.u^{(l)}(k)>u_{d}+\delta\right)$, let $\underline{v}^{(l)}:=\underline{v}^{(l-1)}(k)$ and $\bar{v}^{(l)}:=v^{(l)}(k)$, then go to Step 5 .

Step $6: v^{*}(k):=v^{(l)}(k)$, and stop.

$\Delta=\frac{\alpha_{0}-\beta_{0}}{\mathrm{~L}}$ is the disintegration interval of threshold $\alpha$ and $\beta$, where $\mathrm{L}$ is a sufficient large integer. The calculated input $v^{*}(k)$ is used as the input to the magnetostrictive actuator.

\section{Control experiment}

By using the proposed method, we have performed control experiments to drive the output of the actuator to follow the desired signals.

\subsection{Experiment environment}

In the experiments, the displacement of the actuator is measured with a sensor, and then the measured displacement is converted into a digital signal. By using the digital signal, the input to the actuator is determined so that the output can get closer to the desired one. Fig. 7 shows the experimental environment.

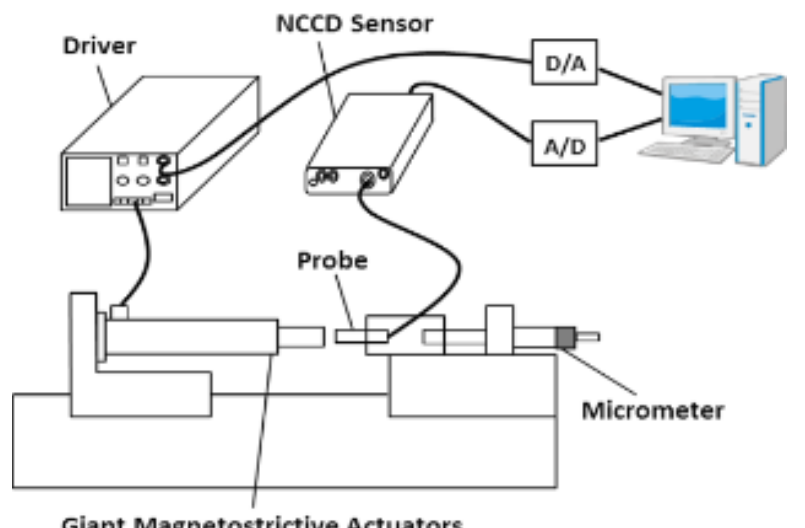

Fig. 7 Experimental environment.

\subsection{Experiment}

For the desired output $u_{d}=5 \sin (2 \pi f \cdot 0.001 k)[\mu m]$, experiments are conducted, where $f$ is the frequency [Hz]. Table 1 shows the experimental results of the tracking errors for different frequencies.

For desired signal with 1Hz, Fig. 8 shows the input signal, Fig. 9 shows the output of the actuator and the desired signal. From Fig. 8 and Fig. 9, it can be seen that the proposed method can control the magnetostrictive actuator well for desired signal with $1 \mathrm{~Hz}$.

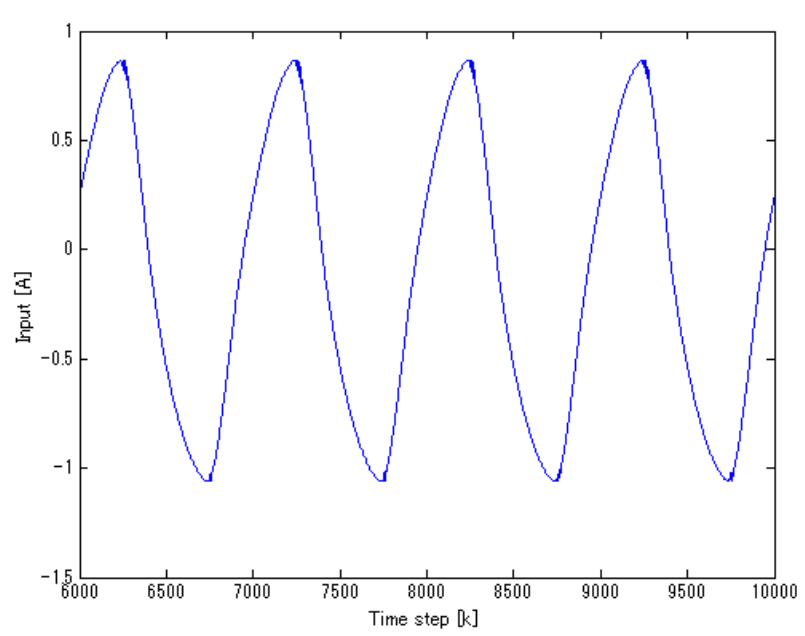

Fig. 8 The input signal for desired signal with $1 \mathrm{~Hz}$.

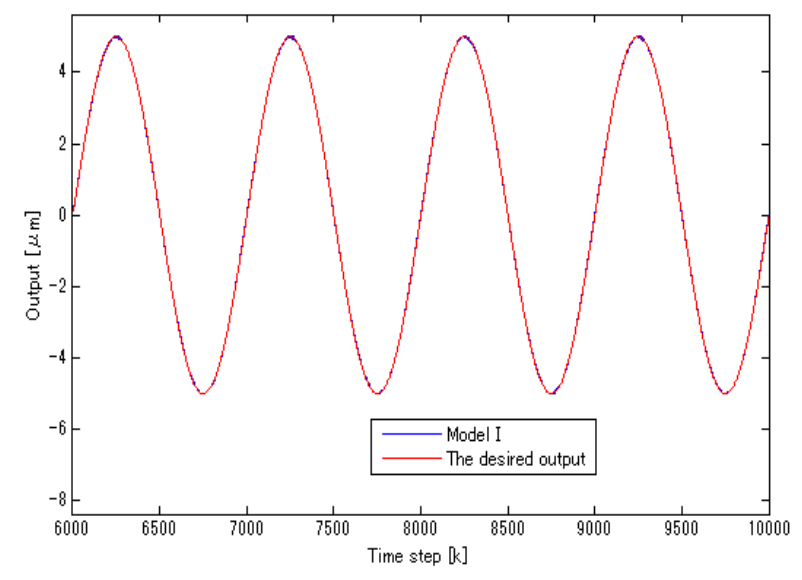

Fig. 9 The controlled output of the actuator and the desired output.

Table 1 Experimental results based on Model I

\begin{tabular}{|c|c|}
\hline Frequency[Hz] & Error $[\boldsymbol{\mu m}]$ \\
\hline \hline 0 & 0.02 \\
\hline 0.2 & 0.04 \\
\hline 1 & 0.05 \\
\hline 5 & 0.08 \\
\hline 10 & 0.10 \\
\hline 20 & 0.21 \\
\hline 30 & 0.61 \\
\hline 40 & 1.00 \\
\hline 50 & 1.40 \\
\hline
\end{tabular}

\begin{tabular}{|c|c|}
\hline Frequency $[\mathrm{Hz}]$ & Error $[\mu \mathrm{m}]$ \\
\hline \hline 60 & 1.82 \\
\hline 70 & 2.08 \\
\hline 80 & 2.75 \\
\hline 90 & 3.10 \\
\hline 100 & 3.68 \\
\hline 110 & 4.44 \\
\hline 120 & 4.66 \\
\hline 130 & 4.52 \\
\hline 150 & 4.60 \\
\hline
\end{tabular}

These results show good performance of the controller in low frequency domain. But the tracking errors for high frequency signals become larger and larger as the frequency increases. Therefore, new control techniques which are effective for high frequency domain is needed to be developed. 


\section{Improved Control Design}

\subsection{Definition of the new model II}

This section proposes a control method corresponding to high frequency domain, where the improvement of the density function is considered since the density function usually depends on the input. Fig. 10 shows the block diagram of this system [8-11]. By observing that the desired output will become the external input of a closed-loop control system, the idea employed here is to include the information of the desired output signal. A more suitable density function is calculated by modeling it using the desired output. By such a method, it is expected that the tracking errors at extreme values and the output tracking for desired signals with high frequencies output may be improved.

By using the desired output values at $\mathrm{k}$-th step and $(\mathrm{k}+1)-$ th step, the density function can be assumed as

$$
\mu(\beta, \alpha, k)=\mu_{1}(\beta, \alpha)+\mu_{2}(\beta, \alpha)\left|u_{d}(k)\right|+\mu_{3}(\beta, \alpha)\left|u_{d}(k+1)\right|
$$

where $\mu_{1}(\beta, \alpha), \mu_{2}(\beta, \alpha), \mu_{3}(\beta, \alpha)$ are non-negative density functions.

The corresponding estimates of $\mu_{i}(\beta, \alpha)$ at step k are assumed as $\hat{\mu}_{i}(\beta, \alpha, k)$ for $\mathrm{i}=1,2,3$, and they are adaptively updated by

$$
\begin{aligned}
& \hat{\mu}_{1}(\beta, \alpha, k)=\left|\hat{\mu}_{1}(\beta, \alpha, k-1)+\frac{\rho}{\left(1+u_{d}{ }^{2}(k)+u_{d}{ }^{2}(k+1)\right) S} e(k-1) \gamma_{\alpha \beta}[v](k-1)\right| \\
& \hat{\mu}_{2}(\beta, \alpha, k)=\left|\hat{\mu}_{2}(\beta, \alpha, k-1)+\frac{\rho\left|u_{d}(k)\right|}{\left(1+u_{d}{ }^{2}(k)+u_{d}{ }^{2}(k+1)\right) S} e(k-1) \gamma_{\alpha \beta}[v](k-1)\right| \\
& \hat{\mu}_{3}(\beta, \alpha, k)=\left|\hat{\mu}_{3}(\beta, \alpha, k-1)+\frac{\rho\left|u_{d}(k+1)\right|}{\left(1+u_{d}{ }^{2}(k)+u_{d}{ }^{2}(k+1)\right) S} e(k-1) \gamma_{\alpha \beta}[v](k-1)\right|
\end{aligned}
$$

Also, the initial values of $\hat{\mu}_{1}(\beta, \alpha, k)$ and $\hat{\mu}_{2}(\beta, \alpha, k)$ and $\hat{\mu}_{3}(\beta, \alpha, k)$ are assumed non-negative.

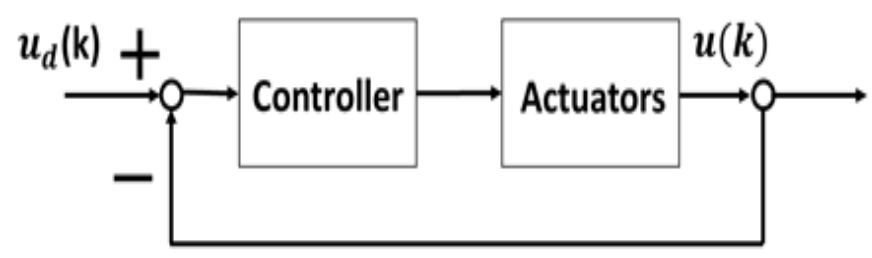

Fig. 10 Block diagram of the closed-loop system.

\subsection{Definition of the model III}

As another model, the density function can be assumed like model II as

$$
\mu(\beta, \alpha, k)=\mu_{1}(\beta, \alpha)+\mu_{2}(\beta, \alpha)\left|u_{d}(k)\right|
$$

where $\mu_{1}(\beta, \alpha), \mu_{2}(\beta, \alpha)$ are non-negative density functions.

This density function only uses the value of desired output signal at step $\mathrm{k}$. Therefore model III has less computational load than model II. The elements $\mu_{1}(\beta, \alpha)$ and $\mu_{2}(\beta, \alpha)$ are adaptively estimated as

$$
\hat{\mu}_{1}(\beta, \alpha, k)=\left|\hat{\mu}_{1}(\beta, \alpha, k-1)+\frac{\rho}{\left(1+u_{d}^{2}(k)\right) S} e(k-1) \gamma_{\alpha \beta}[v](k-1)\right|
$$

$\hat{\mu}_{2}(\beta, \alpha, k)=\left|\hat{\mu}_{2}(\beta, \alpha, k-1)+\frac{\rho\left|u_{d}(k)\right|}{\left(1+u_{d}{ }^{2}(k)\right) S} e(k-1) \gamma_{\alpha \beta}[v](k-1)\right|$

Also, the initial values of $\hat{\mu}_{1}(\beta, \alpha, k)$ and $\hat{\mu}_{2}(\beta, \alpha, k)$ are assumed non-negative.

\subsection{Control design and experimental results}

By using the estimated parameters, the estimated density function can be constructed by using model II and model III. Then, by mimicking the control design method in Section 3, the control input can be derived.

The experimental results show that the tracking error can't be high accuracy. However, it has been observed that the tracking can be improved by using the new models.

As an example, for the desired signal with $100 \mathrm{~Hz}$, Fig. 11 shows the experimental results by using model I and openloop control, Fig. 12 shows the experimental results based on model I and model II. From Fig. 11 and Fig. 12, it can be seen that model II performs better than model I and openloop control, where the tracking at extreme values has been improved because the density function in model II is calculated by using the desired output. Such similar results can be got from $100 \mathrm{~Hz}$ to $150 \mathrm{~Hz}$.

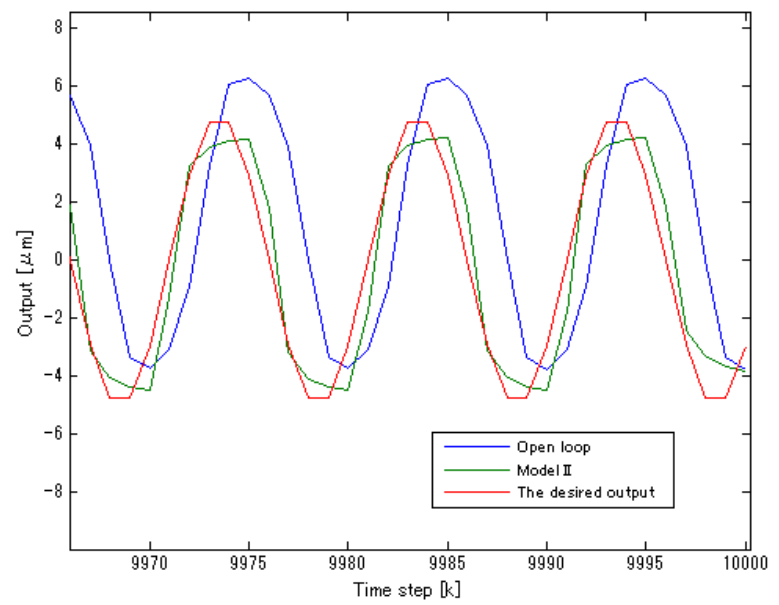

Fig. 11 Experimental results for desired signal with $100 \mathrm{~Hz}$ (1).

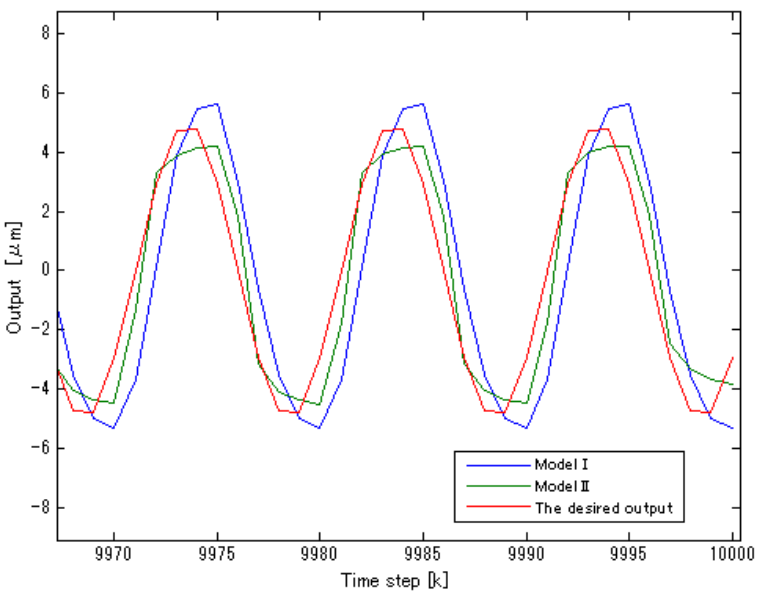

Fig. 12 Experimental results for desired signal with $100 \mathrm{~Hz}$ (2). 
Similarly, the experimental results for desired output with $70 \mathrm{~Hz}$ are shown in Fig. 13 and Fig. 14. Fig. 13 shows the experimental results based on model I and model III, Fig. 14 shows the experimental results based on model II and model III. From Fig. 13 and Fig. 14, it can be seen that model III performs better than model I and model II.

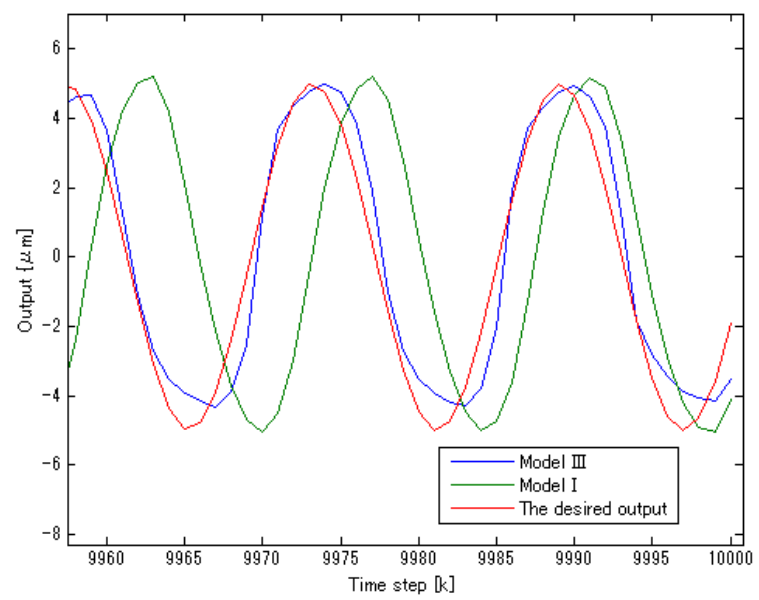

Fig. 13 Experimental results for desired signal with $70 \mathrm{~Hz}$ (1).

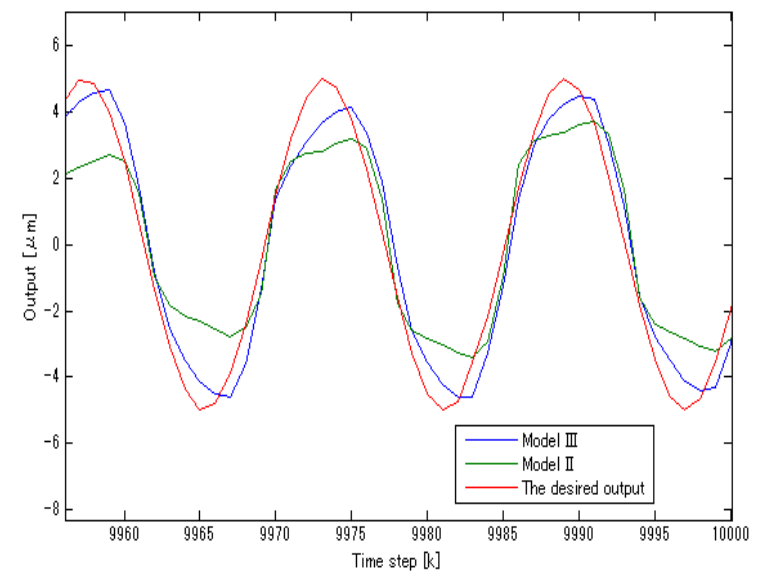

Fig. 14 Experimental results for desired signal with $70 \mathrm{~Hz}$ (2).

Finally, Fig. 15 shows the control experimental results by using Model I in Subsection 3.1, Model II in Subsection 5.1, and Model III in Subsection 5.2.

From Fig. 15, it can be seen that a simple density function can cope with relatively low frequency desired outputs (from $0 \mathrm{~Hz}$ to $30 \mathrm{~Hz}$ ). On the other hand, Model III can cope with the desired outputs with frequencies from $40 \mathrm{~Hz}$ to $90 \mathrm{~Hz}$, whereas Model II can cope with the desired outputs with frequencies from $100 \mathrm{~Hz}$ to $150 \mathrm{~Hz}$.

\section{Conclusions and Discussion}

This paper has considered the control problem for magnetostrictive actuators. Also, the models of density function are enhanced to improve precision for desired outputs with relatively high frequencies. The input-output relations of the magnetostrictive actuator is expressed by using Preisach model, and adaptive control theory is used to control the actuator.

Improved density function models can give a better output tracking than a simple model (model I). Since the tracking error is still large, the proposed models and the control methods are insufficient. In fact, an important factor is considered as the phase difference in the system. Especially, the tracking performance for high frequency desired signals is largely influenced by phase difference. The future research will be focused on the control in consideration of the phase difference.

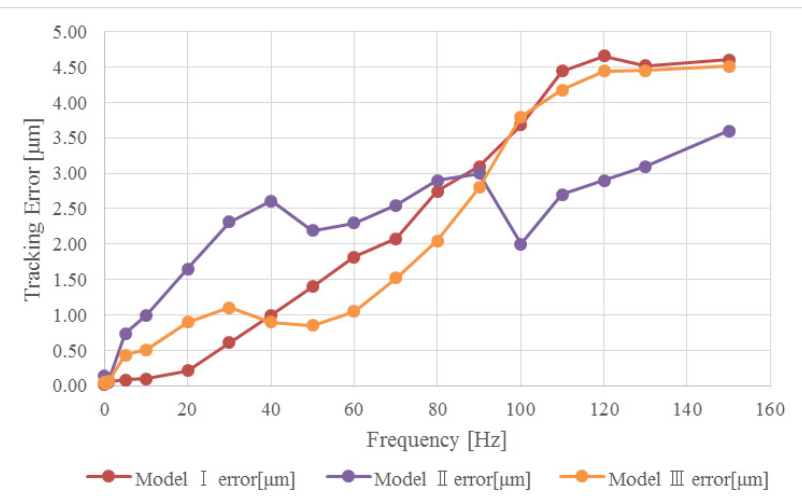

Fig. 15 Experimental results based on different models.

\section{References}

[1] G.C. Goodwin and K.S. Sin, Adaptive Filtering Prediction and Control, Prentice-Hall, 1984.

[2] K.J. Astrom and B. Wittenmark, Adaptive Control, Dover Publications, 2008.

[3] I.D. Mayergoyz, Mathmatical Models of Hysteresis, Springer-Verlag, 1991.

[4] M. Brokate and J. Sprekels, Hysteresis and Phase Transitions, Springer-Verlag, 1996.

[5] B. Widrow and E. Walach, Adaptive Inverse Control, Power Engineering, 1991.

[6] G. Tao, Adaptive Control Design and Analysis, WileyInterscience, 1996.

[7] F. Ikhouane and J. Rodellar Systems with Hysteresis, Wiley, 2007.

[8] K. Shimizu, Feedback control theory, Corona Publishing, 2013.

[9] B. Friedland, Control System design, Dover publications, 2005.

[10] J. Doyle, B. Francis and A. Tannenbum, Feedback Control Theory, Dover publications, 2009.

[11] L. Sciavicco and B. Siciliano, Modelling and Control of Robot Manipulators, Springer-Verlag, 1996.

[12] P. Ioannou and J. Sun, Robust Adaptive Control, Dover publications, 2012.

[13] A. Visintin, Differential Models of Hysteresis, Springer-Verlag, 1994. 